\title{
Automatic Gunn and Salus Sign Quantification in Retinal Images *
}

\author{
Jeffrey Wigdahl, Pedro Guimarães, Georgios Leontidis, Areti Triantafyllou, and Alfredo Ruggeri
}

\begin{abstract}
Prolonged hypertension can lead to abnormal changes in the retinal vasculature, including sclerosis and thickening of the arteriole walls. These changes can cause compression (Gunn's sign) and deflection (Salus's sign) of the veins at arteriovenous crossings. In retinal images, Gunn's sign appears as a tapering of the vein at a crossing point, while Salus's sign presents as an S-shaped curving. This paper presents a method for the automatic quantification of these two signs once a crossover has been detected; combining segmentation, artery vein classification, and morphological feature extraction techniques to calculate vein widths and angles entering and exiting the crossover. The method was tested on a small set of crossings, graded by a set of 3 doctors who were in agreement as having or not having Gunn/Salus sign. Results show separation between the two classes and that we can reliably detect and quantify these sign under the right conditions.
\end{abstract}

\section{INTRODUCTION}

Hypertension affects nearly 70 million American adults and approximately 970 million adults worldwide [1]. Elevated blood pressure puts stress on the body's blood vessels, often causing damage that can lead to a blockage or rupture. These vascular changes also affect the retina, the sum of which are known as hypertensive retinopathy [2]. The focus of this paper is on one specific sign known as arteriovenous (AV) nicking. Thickening of the arteriole wall and/or sclerosis are thought to compress the vein at a crossing point, impeding blood flow, causing a tapering of the vein at the crossing. This tapering is commonly referred to as Gunn's sign. The pressure from the artery can also displace the vein at the crossing, causing the vein to enter and exit the crossing at a $90^{\circ}$ angle. This phenomena is commonly referred to as Salus's sign [3]. Figure 1 shows examples of crossing that show both of the signs.

Crossing abnormalities are a grade 2 sign (1-4 scale) on the Keith-Wegener-Barker hypertensive retinopathy grading scale [4]. To our knowledge, there is no standard for grading the severity of crossing abnormalities. Typically, AV nicking is a binary marker added to the larger hypertensive grading scale. However, AV nicking is a major factor in the development of

*This research was made possible by a Marie Curie grant from the European Commission in the framework of the REVAMMAD ITN (Initial Training Research network), Project number 316990

J. Wigdahl, P. Guimarães, and A. Ruggeri are with the Department of Information Engineering, University of Padova, Via Gradenigo 6/B, Padova, Italy

G. Leontidis is with with the School of Computer Science at the University of Lincoln, LN67TS, Lincoln, UK (tel: +44(0)1522886873, email: gleontidis@lincoln.ac.uk).

A. Triantafyllou is with the $3^{\text {rd }}$ Internal Medicine clinic of Papageorgiou Hospital, Thessaloniki, Greece
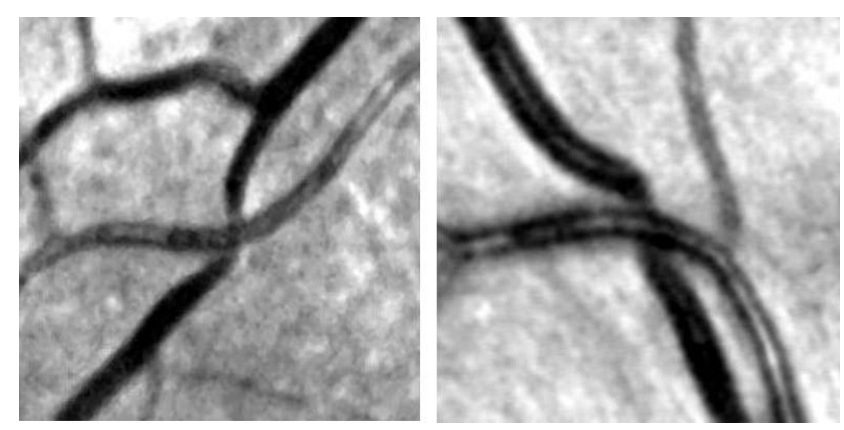

Figure 1: Example images of crossing abnormalities. Left. Image shows both tapering at the crossing (Gunn Sign) and the S shaped curving (Salus Sign). Right. Image shows the $\mathrm{S}$ shaped curving with little to no tapering at the crossing.

branch retinal vein occlusions (BRVO) [5]. Quantification of $\mathrm{AV}$ nicking may be important to the prediction of development to more serious conditions such as this.

In this paper, we present a method for the quantification of the Gunn and Salus sign at retinal arteriovenous crossings. After a crossing is found, the vessels are segmented using the method introduced by Frangi [6]. The four corners of the crossing are detected and used to create bounding box to separate the four vessel segments. Color and intensity are used to determine artery from vein. Vessel widths are detected using monodimensional matched filtering. These widths are used to determine Gunn's sign while the angles created at the corners of the bounding box are used to determine Salus's sign. The results are then compared with the findings of graded images.

\section{DATA DESCRIPTION}

Images were acquired from the 3rd Department of Internal Medicine, Papageorgiou Hospital, Aristotle University of Thessaloniki, Thessaloniki, Greece. A group of three doctors (two hypertension specialists and one ophthalmologist) chose a set of five crossings that they agreed showed both the Gunn and Salus signs, backed by the analysis of fluroangiography images. These five crossing are joined by a set of five normal AV crossings for comparison. Images were captured at a $45^{\circ}$ field of view at $2912 \times 2912 \times 3$ pixels. Images were then cropped to $250 \times 250$ pixels roughly centered on the chosen crossing point. 


\section{Methodology}

This section describes the steps in the Gunn and Salus sign quantification algorithm. The assumption made is that a crossing point has already been found. From the selected crossing point, a 250x250pixel area of interest is cropped from the image. This cropped area is preprocessed by contrast enhancement and illumination correction. A standard morphological top-hat procedure is used which subtracts the morphological opening of image from the original [7]. The images are also blurred using a small Gaussian lowpass filter of size $3 \times 3$ and standard deviation of 1 .

\section{A. Vessel Segmentation}

The well-known multi-scale vessel enhancement method proposed by Frangi [6] is used to segment the vasculature. The eigenvalue decomposition of the Hessian is computed at different scales (convolution with a Guassian of varying sigma values). These scales should cover the width of the structure you are looking to enhance. For this work, the sigma range was from 4-10. The 'vesselness' of a pixel is determined by two metrics known as 'blobness' and 'second order structureness'.

$$
\begin{gathered}
R_{B}=\frac{\lambda_{1}}{\lambda_{2}} \\
S=\sqrt{\lambda_{1}^{2}+\lambda_{2}^{2}}
\end{gathered}
$$

The blobness metric, $\mathrm{R}_{\mathrm{B}}$, is the ratio of the $1^{\text {st }}$ eigenvalue to the $2^{\text {nd }}$. The second order structureness, $\mathrm{S}$, is the square root of the sum of the squared eigenvalues. Vesselness is then determined as:

$$
V_{0}(s c)=\left\{\begin{array}{c}
0, \quad \lambda_{2}=0 \\
e^{\frac{-R_{B}^{2}}{2 \beta^{2}}}\left(1-e^{\frac{s^{2}}{2 c^{2}}}\right)
\end{array}\right.
$$

Where $\beta$ and $\mathrm{c}$ are constants, and sc is the scale. The maximum value is found across all scales, forming a vesselness image. A threshold is applied to the image to create the segmented vessel image after small morphological corrections to fill small holes and omit small disconnected segments.

\section{B. Crossing Segmentation}

Once the vessel segmentation is found, a distance transformation is applied to the binary image with respect to the coordinates of the found crossing point. A search is then performed to find the corners of a bounding box that covers the entire crossing. This is done by finding the closest nonzero valued distance pixel in each direction starting from the crossing coordinates. Lines are projected out from the origin at different angles, the lowest nonzero value for each angle is recorded, and a plot is made of these values. The centers of the four troughs represent the coordinates of the corners of a bounding box covering the crossing. Figure 2 shows an example of this process.

\section{Artery Vein Classification}

Removing the crossover from the vessel segmentation separates the four segments coming in and out of the
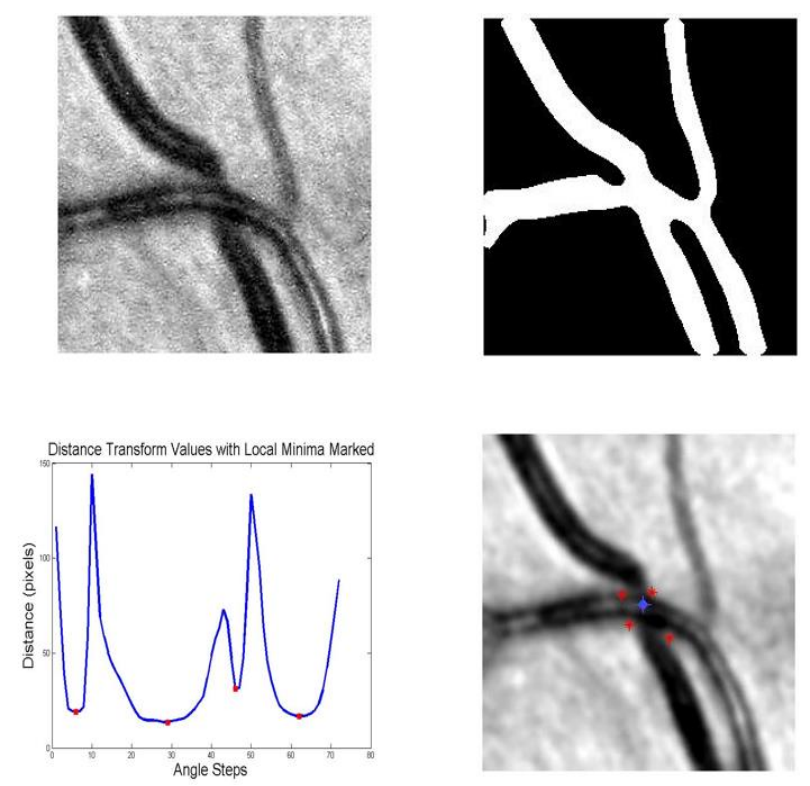

Figure 2: (Top Left) The enhanced green channel image, (Top Right) vessel segmentation after Hessian vessel enhancement, (Bottom Left) graph showing the local minima corresponding to the corners of the crossover, (Bottom Right) and those points on the image along with the selected crossover point.
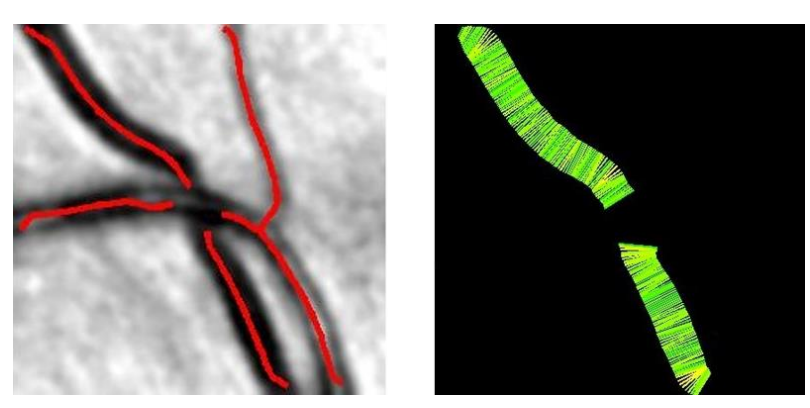

Figure 3: (Left) Graph representation of vessel segments used to determine AV classification. (Right) Found vein segments along with vessel cross sectional lines representing the width and direction at points along the vessel.

crossover. The mean RGB intensity values are calculated for each segment. In general, the arteries are lighter in color and often display a bright central reflex. The segments with the two lowest mean intensities are found as the vein as long as they are across from each other.

\section{Vessel Width Detection}

The vessel segments are skeletonized and converted to a graph representation. Monodimensional matched filters are applied perpendicularly to the profiles along the vessel axis [8]. The borders of the vessel are found as zero points on the enhanced profiles.

\section{E. Gunn and Salus Calculation}

The Gunn sign is a measure of the tapering of the vein at a crossing point. To measure this tapering, an average vessel width value near the crossing and an average value farther from the crossing must be determined. 


$$
g_{i}=\frac{C_{i c}+C_{o c}}{C_{i r}+C_{o r}}
$$

Where $\mathrm{C}_{\mathrm{ic}}$ and $\mathrm{C}_{\mathrm{oc}}$ are the vessel calibers near to the crossing and $\mathrm{C}_{\mathrm{ir}}$ and $\mathrm{C}_{\text {or }}$ are the vessel calibers farther away from the crossing. The mean of the three closest points from the graph representation of the vessel are used for the close caliber calculation, while the rest of the points are used for the far caliber calculation.

The $\mathrm{S}$ shape of the Salus sign can be represented by three angle values. These are the average direction of the two vein segments going in and out of the crossing, and the direction at the crossing.

$$
s_{i}=.5 *\left(a_{1}+a_{3}\right)
$$

Where $\mathrm{a}_{1}$ represents the difference between the angle at the crossing of the vessel with the average angle along the vessel going into the crossing. $a_{3}$ represents these same values leaving the crossing. This average difference is then computed from these values.

The vessel widths necessary for the calculation of the Gunn sign as well as the average direction of each vessel segment have already been calculated in the vessel width detection step. The direction at the crossing is calculated by averaging the points closest to the crossing. For this work, both for the angles and widths of the vein, five points close to the crossing are averaged while 20 points along the remaining length of the vessel are averaged. This is done on both sides of the crossing. Figure 3 shows calculated widths and angles at points along the found vessel.

\section{RESULTS AND DISCUSSION}

To evaluate the algorithm, three graders classified the ten crossings as normal, or showing Gunn/Salus signs. These graders were in agreement on all crossings, removing intergrader variability. The Gunn and Salus sign were then calculated with the proposed algorithm and the results were compared. Table 1 shows the results for each of the ten crossings. The Abnormal crossings had a mean and standard deviation Salus sign of .29+-.06 and a Gunn sign of .78+- .11 . The normal crossings had a mean/standard deviation Salus sign of .19+-.07 and mean Gunn sign of .94 +- .08. The ideal normal crossing would have a Salus sign of 0 and Gunn sign of 1 , with the Salus sign moving higher and the Gunn sign moving lower in the abnormal case. The results show separation between the two groups on this limited data set. However, it is uncertain as to the importance of each individual sign. Whether they can be combined in some manner or thresholded separately. Since the gradings are binary, thresholds should be set for classification purposes. Setting a threshold of .89 for the Gunn sign and .22 for the Salus sign would yield one false positive and one false negative. Figure 4 shows both of these misclassified images along with the most 'abnormal' and 'normal' cases. Even in high quality images, the area around the crossing can have low contrast, leading generally to overestimations of the vessel width at the crossing. This overestimation can also
Table 1: Gunn and Salus calculations for the 5 normal and 5 abnormal crossings.

\begin{tabular}{l|ll}
\hline Crossing & Gunn Sign & Salus Sign \\
\hline NormalCrossing1 & 0.89 & 0.17 \\
NormalCrossing2 & 1.08 & 0.14 \\
NormalCrossing3 & 0.90 & 0.18 \\
NormalCrossing4 & 0.91 & 0.32 \\
NormalCrossing5 & 0.94 & 0.15 \\
AbnormalCrossing1 & 0.71 & 0.39 \\
AbnormalCrossing2 & 0.92 & 0.23 \\
AbnormalCrossing3 & 0.67 & 0.29 \\
AbnormalCrossing4 & 0.88 & 0.27 \\
AbnormalCrossing5 & 0.70 & 0.28 \\
\hline
\end{tabular}

affect the calculated angle into the crossing as well. For this reason, a small buffer is used to avoid the closest points to the crossing. For these calculations, the first 3 points closest to the crossing are discarded. However, it is still likely that the misclassified images suffered from an overestimation of the width at the crossing.

There are other factors that can contribute to errors in the calculations. The most important and likely is an error in the vessel segmentation. Generally this is attributed to the quality of the image. Another factor is the angle at which the crossing takes place. If the vein and artery are nearly parallel, it is difficult for segmentation algorithms to differentiate between the vessels, which can also lead to segmentation errors. One other likely segmentation error can come from a strong central reflex on the artery.

The algorithm runs a single image using a single core MATLAB (The Mathworks Inc., Natick, MA) implementation in $3.4+-.3 \mathrm{~s}$ on an Intel Core i7-4770 CPU (Intel Corporation, Santa Clara, CA) at $3.4 \mathrm{GHz}$.

\section{CONCLUSIONS}

This paper presents a method for the automatic quantification of the Gunn and Salus sign in retinal images. Results on a set of ten crossings showed separation between the two classes and agreement with the manual graders. Despite the small sample size, the results are promising and warrant testing on a larger data set. There is still a lack of knowledge as to the importance of the degree or severity of these signs. As of now, they are a binary classification for an ophthalmologist, but future studies may reveal the importance of Gunn/Salus scale, or whether one sign is a better predictor for BRVO and other advance hypertensive retinopathy signs. The quality and resolution of the retinal images used has made this analysis possible. Although no test was done, it is very likely that this method would fail under lesser conditions.

Improvements to the algorithm could be seen by automatically detecting the crossovers. There are many methods to do this $[9,10]$, but this would have added another 

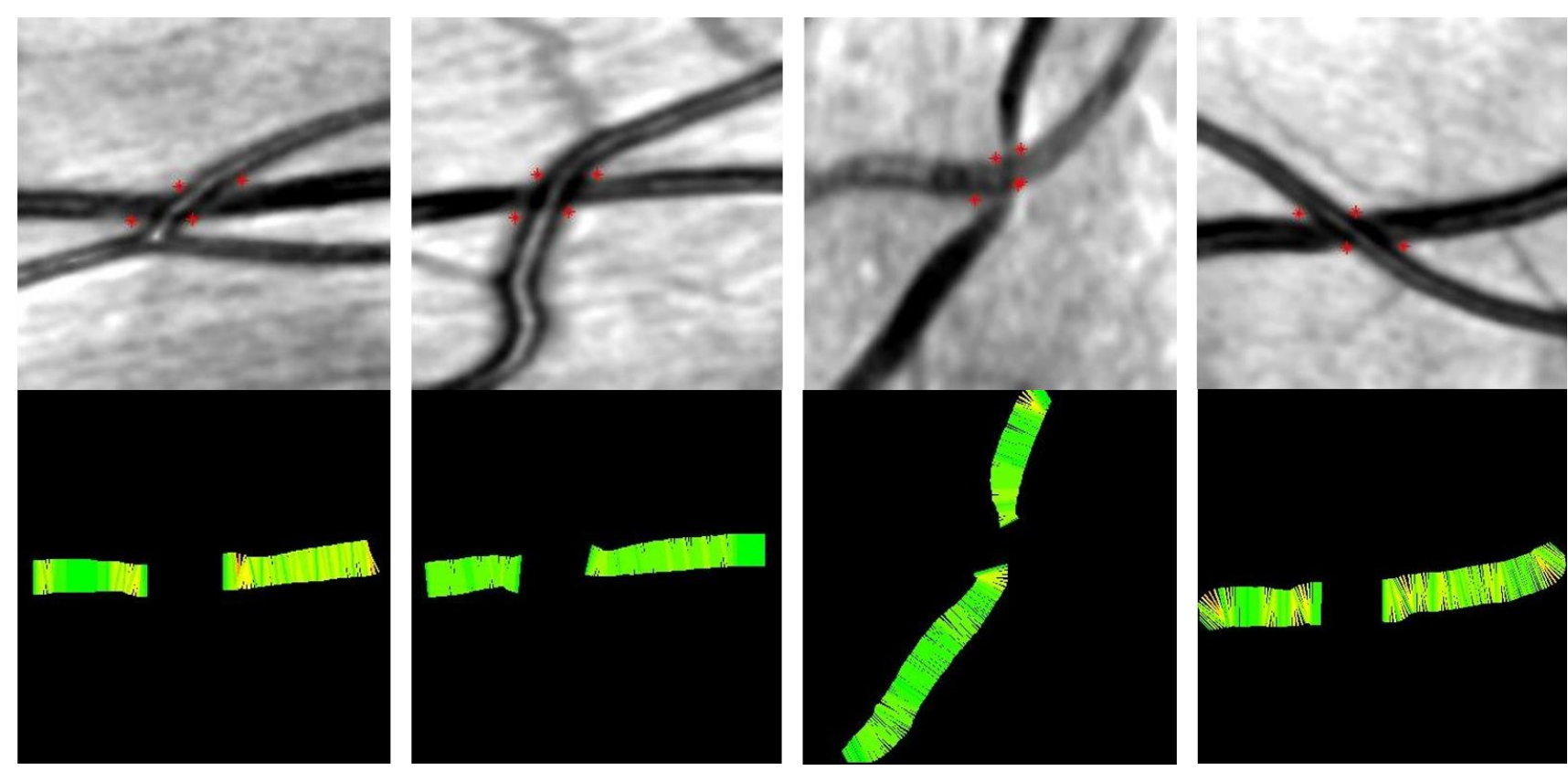

Figure 4: Each column shows the original image and the segmented vein widths and angles at points along the vessels (1) Abnormal crossing 2 which had a Gunn sign close to 1.(2) Normal crossing 4 which had an elevated Salus sign.(3) Abnormal crossing 3 (4) Normal crossing 2 .

layer of error to deal with, while the accuracy of the Gunn and Salus sign calculation was the main goal of this paper. Another improvement would be to add an image quality module to ensure the crossover is of sufficient quality before quantification is attempted.

Future work would include testing the algorithm on a larger data set. The graders could also be asked to rank the Gunn and Salus sign at a crossing compared with the other images in the data set. A rank correlation between the graders and algorithm could then be established, providing stronger evidence of agreement with human observation.

\section{ACKNOWLEDGMENTS}

The Authors would like to thank Panagiotis Symeonidis and Stella Douma from Papageorgiou Hospital, Thessaloniki, Greece for providing the images and their expertise in the grading of the crossovers.

\section{REFERENCES}

[1] Nwankwo T, Yoon SS, Burt V, Gu Q. Hypertension among adults in the US: National Health and Nutrition Examination Survey, 2011-2012. NCHS Data Brief, No. 133. Hyattsville, MD: National Center for Health Statistics, Centers for Disease Control and Prevention, US Dept of Health and Human Services; 2013.

[2] Tso M, Jampol LM (1982) Pathophysiology of hypertensive retinopathy.Ophthalmology, 89, 1132-45.

[3] Patel V, Kohner EM. Eye in hypertension. In: Swales JD (ed) Text Book of Hypertension Blackwell Scientific Publications: Oxford, 1994, pp 1015-1025.

[4] Keith NM, Wagener HP, Barker NW. Some different types of essential hypertension: their course and prognosis. Am J Med Sci 1939;197:332343.

[5] Klein R, Klein BE, Moss SE, Meuer SM. The epidemiology of retinal vein occlusion: the Beaver Dam Eye Study. Trans Am Ophthalmol Soc 2000; 98: 133-41.
[6] Frangi, A.F., Niessen, W.J., Vincken, K.L., Viergever, M.A., 1998 Multiscale vessel enhancement filtering. Proc. Int. Conf. Medical Image Computing Computer-Assisted Intervention. Lect. Notes Comp. Sci. 1496, 130-137.

[7] Soille Pierre. Morphological image analysis: principles and applications. Springer-Verlag New York, Inc. 2003

[8] C. Tomasi and R. Manduchi, "Bilateral filtering for gray and color images," in Pro-ceedings 6th International Conference on Computer Vision, Bombay, India, Janu-ary 1998, pp. 839-846.

[9] Calvo, D., Ortega, M., Penedo, M.G., Rouco, J., 2011. Automatic detection and characterisation of retinal vessel tree bifurcations and crossovers in eye fundus images. Comput. Methods Prog. Biomed. 103 (1), 28-38.

[10] Bhuiyan, A., Nath, B., Chua, J., Ramamohanarao, K., 2007. Automatic detection of vascular bifurcations and crossovers from color retinal fundus images. In: Third Internat. IEEE Conf. on Signal-Image Technologies and Internet-Based System (SITIS), pp. 711-718. 